\title{
Evaluación educacional a las tecnologías de información y comunicación con modelos estadísticos
}

\section{Educational assessment of information and communication technologies through statistical models}

Oswaldo Villacrés Cáceres. ${ }^{1}$, Juan Carlos Fiallos Quinteros. ${ }^{2}$, Héctor Mullo Guaminga. ${ }^{3}$ \& Stefany Diaz Andrade. ${ }^{4}$

\begin{abstract}
.
DOI: https://doi.org/10.33262/concienciadigital.v3i3.1.1384

This work aims to present the impact evaluation carried out to determine the scope of 'Information and Communication Technologies' usage and its incidence on academic achievements regarding the pilot project "Cognitive Tutoring System for secondary education in Ecuador", implemented by Escuela Superior Politécnica del Litoral. In order to conduct this study, the Statistical Model was applied, and the data was obtained through a quasi-experimental design. The results therefore allow establishing ITC effect on math performance of eighth grade students from the urban-marginal schools in Guayaquil, which took part in this study. In conclusion, the pilot study 'Cognitive Tutor System' allowed increasing by 0.54 points the average Math score; consequently, it aided to reduce the knowledge gap of the sample group.
\end{abstract}

Keywords Educational Measurement, Information Technology, Statistical Models.

\section{Resumen.}

El artículo pretende exponer la evaluación de impacto realizada para determinar el nivel de incidencia de las Tecnologías de Información y Comunicación, y los resultados alcanzados en el rendimiento académico con el estudio piloto "Sistema de Tutoría Cognitiva para educación secundaria en Ecuador", proyecto implementado por la Escuela Superior Politécnica del Litoral. Para su desarrollo, se especifica el modelo estadístico y los resultados alcanzados a lo largo de un estudio de diseño cuasiexperimental. Los resultados permiten establecer su efecto sobre el rendimiento académico en la asignatura de matemática en estudiantes de octavo de educación básica de Unidades Educativas

\footnotetext{
${ }^{1}$ Escuela Superior Politécnica de Chimborazo, Facultad de Ciencias, Riobamba, Ecuador. ovillacres@espoch.edu.ec

${ }^{2}$ Universidad Nacional de Colombia, Bogotá, Colombia. juank.fiallos@gmail.com

${ }^{3}$ Escuela Superior Politécnica de Chimborazo, Facultad de Ciencias, Riobamba, Ecuador. hmullo@espoch.edu.ec

${ }^{4}$ Escuela Superior Politécnica de Chimborazo, Facultad de Ciencias, Riobamba, Ecuador. stefany.diaz@espoch.edu.ec
} 
ubicadas en zonas urbano-marginales de la ciudad de Guayaquil que colaboraron en el estudio piloto. En conclusión, estudio piloto Sistema de Tutor Cognitivo tiene un impacto de 0,54 puntos en la nota promedio de la asignatura de matemática, logrando disminuir al menos en la muestra estudiada la brecha de conocimiento en los estudiantes del grupo de tratamiento.

Palabras Claves Evaluación Educacional, Tecnología de la Información, Modelos Estadísticos.

\section{Introducción.}

Todas las teorías del desarrollo han admitido que el progreso de las naciones tiene como arista principal a la educación, tanto por el mejoramiento como por la adquisición de nuevas capacidades de las personas o simplemente por el incremento del capital humano. Para que exista un efecto fortalecedor en las personas, permitiéndoles desarrollarse completamente y lograr el bienestar, la educación debe ser de calidad. (Hanushek et al., 2007) La educación es un derecho fundamental de los seres humanos ("Naciones Unidas", 2015) y para que se vea reflejada en varios aspectos de la sociedad, corresponde a los Estados garantizar el acceso y la calidad de la educación considerando su relevancia, pertinencia y equidad. Para Atilio Pizarro ("Semana", 2017), jefe de Planificación de la UNESCO durante la XV asamblea anual de la Fundación Empresarios por la Educación, la educación debe ser de calidad, formando estudiantes con habilidades que permitan enfrentar los desafíos del mundo de hoy, sin que nadie sea excluido.

En América Latina se ha evidenciado una baja calidad de la educación de acuerdo a los resultados del Programa para la Evaluación Internacional de los Alumnos (PISA, por sus siglas en inglés), considerando que el puntaje promedio alcanzado por los países de la Organización para la Cooperación y el Desarrollo Económicos (OCDE) es de 487 puntos sobre 500, todos los países latinoamericanos, evaluados se encuentran por debajo de este valor promedio, incluidos Chile y México, países pertenecientes a la OCDE ("BBC News Mundo", 2019) lo cual genera brechas económicas y sociales, que profundizan la desigualdad entre las personas, las comunidades, países y regiones (Pérez, 2019). Según el análisis realizado por la UNESCO, y de acuerdo a los resultados de sus evaluaciones en lo que respecta a acceso, continuidad de estudios y logros de aprendizaje, sigue existiendo desigualdad educativa en los países latinoamericanos. Para mejorar la calidad de la educación, las naciones han invertido considerables recursos económicos en la generación de políticas, programas y proyectos articulados, como también en la dotación de infraestructura, diseños o actualizaciones curriculares, entre otros; sin que hayan causado los cambios deseados.

Para alcanzar los cambios deseados, en pro de mejorar la calidad de la educación, los países de América Latina a mediados de los años noventa integran recursos y herramientas tecnológicas en el proceso enseñanza-aprendizaje. Por citar algunos resultados alcanzados, en la investigación desarrollada por Alcibar, Monroy y Jiménez (2018), han observado que los estudiantes del Instituto Politécnico Nacional de la Ciudad de México, usan herramientas 
informáticas por lo menos una vez a la semana como medio de consulta para la realización de tareas escolares, concluyendo que aspectos como la computadora, la forma amigable y divertida de hacer las tareas por internet influyen de forma positiva en el aprendizaje. Mientras, Alderete y Formichella (2016) concluyeron en su estudio que las TIC tanto en el ámbito del hogar como en la escuela, contribuyen a mejorar el rendimiento de los estudiantes. Para Granados Ospina (2015), el uso de las TIC supone romper con los medio tradicionales, pizarra, marcador, borrador entre otros; para dar paso a la función docente, basada en la necesidad de formarse y actualizar sus métodos en función de los requisitos actuales.

"En el 2006 es cuando Ecuador formalmente se afianza en incorporar las TIC a la gestión pública y a los procesos educacionales", principalmente en la "dotación de infraestructura, equipamiento de aulas con ordenadores y recursos informáticos, dotación de software educativo, capacitación al profesorado, creación de portales educativos, soporte técnico a las escuelas, entre otros." (Peñaherrera León, 2012). Sin embargo, la evaluación de los resultados de estas iniciativas ha sido insuficiente, dado que en muchos casos su implementación ha resultado poco rigurosa e intuitiva, pero además se relaciona con la falta de instrumentos específicos que den confianza para medir estos impactos, separándolos adecuadamente de otras innumerables variables presentes en los procesos educativos, y que son afectadas dinámicamente con la introducción de TIC. (Severin, 2010, citado en Fiallos, 2014)

En Ecuador, en 2008, la Asamblea Nacional aprueba una nueva constitución; en el Título VII: Régimen del buen vivir, sección Educación, artículo 347, inciso octavo, establece que será responsabilidad del Estado: "Incorporar las tecnologías de la información y comunicación en el proceso educativo y propiciar el enlace de la enseñanza con las actividades productivas o sociales." (Asamblea Nacional Constituyente del Ecuador, 2008) Asimismo, el Plan Nacional de Desarrollo o Plan Nacional para el Buen Vivir 2009-2013, establece como Objetivo Nacional, en su Política 2.7 apartado a: "Democratizar el acceso a las tecnologías de información y comunicación, especialmente a Internet, a través de la dotación planificada de infraestructura y servicios necesarios a los establecimientos educativos públicos de todos los niveles y la implantación de telecentros en las áreas rurales." (Secretaría Nacional de Planificación y Desarrollo - SENPLADES, 2009)

A partir de las disposiciones anteriores, se han desarrollado diversidad de proyectos que incluyen la incorporación de TIC en el sistema educativo.

Considerando que la función de un tutor virtual es similar a la de un profesor en modalidad presencial, es decir, asistir o guiar al estudiante en su proceso formativo para alcanzar los logros de aprendizaje y que permite vencer las barreras de espacio y tiempo (Espinoza Freire \& Ricaldi Echevarría, 2018); la Escuela Superior Politécnica del Litoral (ESPOL) desarrollo el estudio "Sistemas de Tutoría Cognitiva" con la participación de estudiantes de octavo de básica de unidades educativas en el Ecuador, con el financiamiento del Banco Interamericano de Desarrollo (BID). En el estudio piloto se trazó como objetivo primordial el mejorar el 
desempeño académico en la asignatura de matemática a nivel secundario de unidades educativas mediante la integración de recursos y herramientas tecnológicas, para lo cual, se llevaron a cabo: implementación de un sistema informático denominado Tutor Cognitivo, capacitación a profesores, contenido adaptado a las necesidades locales, valoración del desempeño docente y al rendimiento académico de los estudiantes en la asignatura de matemática.

En este contexto, el presente artículo pretende determinar el impacto que tiene el proyecto con la incorporación del Sistema de Tutor Cognitivo como elemento mediador en el proceso enseñanza-aprendizaje y cuantificar su incidencia en el desempeño académico de los estudiantes de la asignatura de matemática, mediante un análisis estadístico basado en los resultados de pruebas estandarizadas aplicadas en la misma.

\section{Metodología.}

El principal objetivo es determinar el nivel de impacto a través de un modelo estadístico que permita cuantificar los efectos de las herramientas y recursos tecnológicos TICs en el desempeño académico, como parte del proyecto piloto Sistema de Tutoría Cognitiva desarrollado por la Escuela Superior Politécnica del Litoral en estudiantes de octavo de básica de unidades educativas de Guayaquil.

Se utiliza una metodología cuasi-experimental, para lo cual, se definieron dos grupos de estudio (tratamiento y control). Para el grupo de tratamiento se incorporó el Sistema de Tutor Cognitivo en el proceso enseñanza-aprendizaje, mientras que, en el grupo de control se mantiene el modelo educacional tradicional.

Para determinar los avances en conocimientos en Matemática, el desarrollo del estudio utiliza la fuente de datos obtenida a través de una evaluación realizada en dos tomas (una como línea base y una de seguimiento) a estudiantes de 6 escuelas urbano-marginales de la ciudad de Guayaquil, ver Tabla 1. La toma de línea base para determinar el desempeño de los estudiantes antes de las acciones planificadas y la toma de seguimiento para determinar su desempeño una vez utilizó el Sistema de Tutor Cognitivo. En cada unidad educativa se contó con dos paralelos de octavo de básica, de lo cual, se estableció una dentro del grupo de tratamiento y otro en el grupo de control, ver

Tabla 2. La muestra analizada en las dos tomas es de 396 estudiantes (190 del grupo de tratamiento y 206 del grupo de control).

Tabla 1: Tomas, grupos y muestra de estudio

\section{Total}

\begin{tabular}{lllll} 
Grupo & $\begin{array}{l}\text { Línea } \\
\text { Base }\end{array}$ & Seguimiento & & \\
& & & Cant. & $\%$ \\
\hline Control & 206 & 206 & 412 & $52 \%$ \\
\hline
\end{tabular}




\begin{tabular}{lllll}
\hline Tratamiento & 190 & 190 & 380 & $48 \%$ \\
Total & 396 & 396 & 792 & $100 \%$ \\
\hline
\end{tabular}

Elaborado: Autores

Fuente: Proyecto Tutor Cognitivo

Tabla 2: Número de estudiantes por escuela participante y grupo

\begin{tabular}{llll}
\hline & \multicolumn{2}{l}{ Grupo } & \multirow{2}{*}{ Total } \\
\cline { 2 - 3 } Escuela (Unidad Educativa) & Control & Tratamiento & \\
U. E. del Milenio J.J. Castelló Zambrano & 19 & 19 & 38 \\
U. E. Remigio Romero & 38 & 30 & 68 \\
U. E. Alfredo Portaluppi & 38 & 36 & 74 \\
U. E. Gral. Luis Molina Arroyo & 48 & 48 & 96 \\
U. E. del Milenio Alfredo Vera Vera & 35 & 31 & 66 \\
U. E. Bernardino Echeverría & 28 & 26 & 54 \\
Total general & $\mathbf{2 0 6}$ & $\mathbf{1 9 0}$ & $\mathbf{3 9 6}$ \\
\hline
\end{tabular}

Elaborado: Autores

Fuente: Proyecto Tutor Cognitivo

Para dar seguimiento al desempeño académico de los estudiantes se aplicó una evaluación estándar con 10 unidades de estudio, cada unidad con una puntuación de uno (1) si había sido comprendida por los estudiantes y cero (0) si no fue comprendida. Se totalizaron los resultados de cada capítulo para obtener un Puntaje Total por cada estudiante.

Puesto que se trata de un diseño cuasi-experimental, en el que no se puede asegurar que la variable de impacto (nota promedio de los estudiantes) antes de la intervención sea igual para los dos grupos se utiliza el estimador de diferencias en diferencias. Para medir el impacto del proyecto Tutor Cognitivo, se utiliza el siguiente modelo estadístico:

(1)

$$
y_{i}=\mu+\lambda \cdot T_{i}+\omega \cdot G_{i}+\alpha\left(T_{i} \cdot G_{i}\right)+\varepsilon_{i}
$$

En donde:

$y_{i}$, variable dependiente, nota promedio por cada i-estudiante. 
$T$, variable dicotómica, corresponde a la toma (0 línea base y 1 seguimiento).

$G$, variable dicotómica, corresponde al grupo ( 0 control y 1 tratamiento).

$T \cdot G$, interacción entre tipo de toma (línea base y seguimiento) y grupo de estudio (control y tratamiento).

$\varepsilon_{i}$, error con media cero independiente e idénticamente distribuido.

De lo cual, analizando el modelo de acuerdo a los diferentes valores de las dos variables dicotómicas:

$\mu$, grupo de control en línea base.

$\mu+\lambda$, grupo de control en seguimiento.

$\mu+\omega$, grupo de tratamiento en línea base.

$\mu+\lambda+\omega+\alpha$, grupo de tratamiento en seguimiento.

También, para cuantificar el impacto del proyecto considerando aspectos del entorno en donde se desenvuelven los estudiantes y/o características particulares a cada estudiante, es decir, covariantes, se utiliza el siguiente modelo:

(2)

$$
y_{i}=\mu+\lambda \cdot T_{i}+\omega \cdot G_{i}+\alpha\left(T_{i} \cdot G_{i}\right)+\beta_{k} X_{k i}+\varepsilon_{i}
$$

En donde:

$X_{k i}$, k-ésima covariable que se introduce en el modelo.

$\beta_{k}$, coeficiente de la k-esima covariable.

Finalmente, se calculará el Tamaño del efecto, que nos indica cuánto de la variable dependiente se puede controlar, predecir o explicar por la variable independiente. (Snyder y Lawson, 1993, en Morales, 2012, citado en Fiallos, 2014).

En donde:

$$
d=\frac{X_{\text {post-test Trat }}-X_{\text {post-test Cont }}}{\sigma_{\text {post-test Cont }}}
$$

$d$, tamaño del efecto.

$X_{\text {post-test Trat }}$, media de las notas del grupo de tratamiento en la toma de seguimiento. 
$X_{\text {post-test Cont }}$, media de las notas del grupo de control en la toma de seguimiento.

$\sigma_{\text {post-test cont }}$, desviación para el grupo de control en la toma de seguimiento.

\section{Resultados}

A continuación, se detallan los principales resultados:

La Tabla 3, presenta el número de estudiantes categorizados por sexo y por grupo de tratamiento, de lo cual, se observa en el grupo de control la existencia de una proporción similar de hombres y mujeres. Mientras el grupo de tratamiento tiene mayor porcentaje de hombres $(56,80 \%)$ que de mujeres $(43,20 \%)$

Tabla 3: Número estudiantes por sexo y grupo

\begin{tabular}{|c|c|c|c|c|c|c|}
\hline \multirow{3}{*}{ Sexo } & \multicolumn{6}{|c|}{ Grupo } \\
\hline & \multicolumn{2}{|c|}{ Control } & \multicolumn{2}{|c|}{ Tratamiento } & \multicolumn{2}{|l|}{ Total } \\
\hline & Cant. & $\%$ & Cant. & $\%$ & Cant. & $\%$ \\
\hline Masculino & 107 & 51,90 & 108 & 56,80 & 215 & 54,30 \\
\hline Femenino & 99 & 48,10 & 82 & 43,20 & 181 & 45,70 \\
\hline Total & 206 & 100 & 190 & 100 & 396 & 100 \\
\hline
\end{tabular}

Elaborado: Autores

Fuente: Proyecto Tutor Cognitivo

El Gráfico 1, detalla la nota promedio alcanzada por el grupo de control y tratamiento, en la toma de Línea Base. De lo cual, se concluye que la nota promedio entre los dos grupos no presenta mayor diferencia, por otra parte, la desviación típica hace notar que existe mayor dispersión entre las notas del grupo de tratamiento.

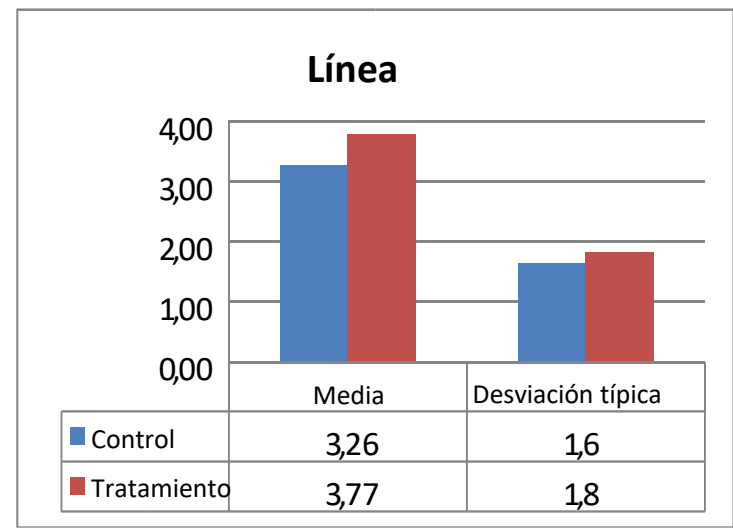

Gráfico 1: Nota promedio por grupo, toma Línea Base. 
En el Gráfico 2, se precisa la nota promedio alcanzada por los dos grupos en cuestión, en la Toma de Seguimiento. De lo cual, se destaca que la nota promedio en el grupo de tratamiento incrementó 1,05 respecto del grupo de control. Además, la desviación típica en el grupo de tratamiento es inferior al grupo de control, lo que indica que las notas obtenidas por los estudiantes tienen mayor homogeneidad respecto de la nota promedio. En consecuencia, el Tutor Cognitivo logra disminuir al menos en la muestra estudiada la brecha de conocimiento entre los estudiantes del grupo de tratamiento.

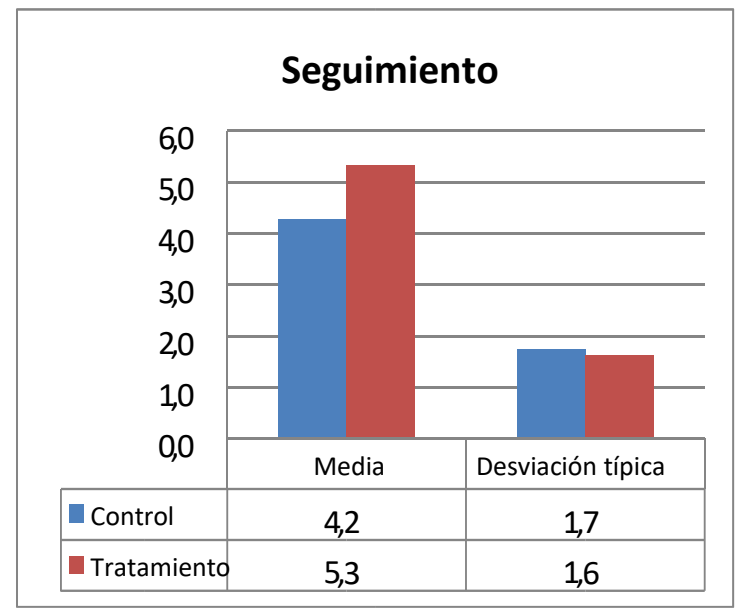

Gráfico 2: Nota promedio por grupo, toma Seguimiento.

A continuación, se analizan los resultados obtenidos al utilizar algunos modelos estadísticos, de acuerdo con las ecuaciones (1) y (2).

\section{Modelo 1}

Para el primer modelo, se consideran únicamente la toma y el grupo, es decir, se utiliza la ecuación (1).

$y_{i}=\mu+\lambda \cdot T_{i}+\omega \cdot G_{i}+\alpha\left(T_{i} \cdot G_{i}\right)+\varepsilon_{i}$,

De lo cual, R-square $=0,16517$.

Tabla 4: Resultados Modelo 1.

\begin{tabular}{|c|c|c|c|c|c|c|c|}
\hline \multirow{2}{*}{ Variable } & \multicolumn{3}{|c|}{ Línea Base } & \multicolumn{3}{|c|}{ Seguimiento } & \multirow{2}{*}{$\begin{array}{l}\text { Diferencias } \\
\text { en } \\
\text { diferencias }\end{array}$} \\
\hline & Control & Tratamiento & Diferencia & Control & Tratamiento & Diferencia & \\
\hline Nota & 3.262 & 3.774 & 0.512 & 4.277 & 5.326 & 1.05 & 0.538 \\
\hline Std. Error & 0.119 & 0.124 & 0.172 & 0.119 & 0.124 & 0.172 & 0.243 \\
\hline $\mathrm{t}$ & 27.39 & 7.39 & 2.98 & 11.78 & 9.13 & 3.64 & 2.21 \\
\hline $\mathrm{P}>\mathrm{t}$ & 0 & 0 & $0.003 * * *$ & 0 & 0 & $0.000 * * *$ & $0.027 * *$ \\
\hline
\end{tabular}


* Medias y Errores Estándar son estimados por regresión lineal

**Inferencia: $* * * \mathrm{p}<0.01 ; * * \mathrm{p}<0.05 ; * \mathrm{p}<0.1$

Elaborado: Autores

Como consecuencia, se evidencia que los coeficientes son estadísticamente significativos, por tanto, se encuentra que el Sistema de Tutor cognitivo tiene un impacto de 0,54 sobre la nota promedio, con error estándar de 0,24.

\section{Modelo 2}

Para el segundo modelo, se incorporan covariables, para este caso la variable escuela (como variable dicotómica) a la que pertenece el estudiante. Se utiliza la ecuación (2), considerando la variable mencionada anteriormente:

$y_{i}=\mu+\lambda \cdot T_{i}+\omega \cdot G_{i}+\alpha\left(T_{i} \cdot G_{i}\right)+\beta_{k} X_{k i}+\varepsilon_{i}$ con $\mathrm{k}=1,2,3,4,5,6$

De lo cual, R-square $=0,31379$

Tabla 5: Resultados coeficientes covariables con Modelo 2

\begin{tabular}{lllll}
\hline Covariables & Coeficiente & Std. Err. & $\mathrm{t}$ & $\mathbf{P}>\mathbf{t}$ \\
\hline Escuela 1 & 0 & 0 & $\cdot$ & $\cdot$ \\
Escuela 2 & -0.016 & 0.223 & -0.07 & 0.944 \\
Escuela 3 & -0.433 & 0.219 & -1.972 & 0.049 \\
Escuela 4 & -0.728 & 0.211 & -3.453 & 0.001 \\
Escuela 5 & 0.978 & 0.224 & 4.367 & 0 \\
Escuela 6 & 1.21 & 0.233 & 5.197 & 0 \\
\hline
\end{tabular}

Tabla 6: Resultados Modelo 2

\begin{tabular}{llllllll}
\hline \multirow{2}{*}{ Variable } & \multicolumn{2}{l}{ Línea Base } & \multicolumn{4}{l}{ Seguimiento } & \multicolumn{2}{c}{$\begin{array}{l}\text { Diferencias } \\
\text { en }\end{array}$} \\
\cline { 2 - 8 } & Control & Tratamiento & Diferencia & Control & Tratamiento & Diferencia \\
diferencias
\end{tabular}

* Medias y Errores Estándar son estimados por regresión lineal

$* *$ Inferencia: $* * * \mathrm{p}<0.01 ; * * \mathrm{p}<0.05 ; * \mathrm{p}<0.1$

Elaborado: Autores 
De los resultados, los valores para los coeficientes de las covariables: Escuela 1 y Escuela 2, no son significativos. Destacar, con el modelo 2 se sigue manteniendo el impacto en la nota promedio de 0,54 .

\section{Modelo 3}

En el tercer modelo, se consideran las covariables: sexo, edad y escuela (excluyendo la 1 y 2 , que fueron no significativos, según el modelo 2).

$y_{i}=\mu+\lambda \cdot T_{i}+\omega \cdot G_{i}+\alpha\left(T_{i} \cdot G_{i}\right)+\beta_{k} X_{k i}+\operatorname{sexo}+$ edad $+\varepsilon_{i}$ con $\mathrm{k}=3,4,5,6$

De lo cual, R-square $=0,31611$

Tabla 7: Resultados coeficientes covariables con Modelo 3

\begin{tabular}{lllll}
\hline Covariables & Coeff. Std. & Err. & $\mathbf{t}$ & $\mathbf{P}>\mathbf{t}$ \\
\hline Escuela 3 & -0.479 & 0.17 & -2.815 & 0.005 \\
Escuela 4 & -0.766 & 0.158 & -4.858 & 0 \\
Escuela 5 & 0.964 & 0.174 & 5.55 & 0 \\
Escuela 6 & 1.221 & 0.193 & 6.316 & 0 \\
Sexo & -0.01 & 0.12 & -0.086 & 0.931 \\
Edad & 0.106 & 0.066 & 1.608 & 0.108 \\
\hline
\end{tabular}

Tabla 8: Resultados Modelo 3

\begin{tabular}{|c|c|c|c|c|c|c|c|}
\hline \multirow{2}{*}{ Variable } & \multicolumn{3}{|c|}{ Línea Base } & \multicolumn{3}{|c|}{ Seguimiento } & \multirow{2}{*}{$\begin{array}{l}\text { Diferencias } \\
\text { en } \\
\text { diferencias }\end{array}$} \\
\hline & Control & Tratamiento & Diferencia & Control & Tratamiento & Diferencia & \\
\hline Nota & 1.964 & 2.462 & 0.498 & 2.979 & 4.015 & 1.036 & 0.538 \\
\hline $\begin{array}{l}\text { Std. } \\
\text { Error }\end{array}$ & 0.778 & 0.798 & 0.158 & 0.778 & 0.798 & 0.158 & 0.221 \\
\hline $\mathrm{t}$ & 2.53 & 2.59 & 3.16 & 3.27 & 4.15 & 3.91 & 2.44 \\
\hline$P>t$ & 0.012 & 0.002 & $0.002 * * *$ & 0 & 0 & $0.000 * * *$ & $0.015 * *$ \\
\hline
\end{tabular}

* Medias y Errores Estándar son estimados por regresión lineal

$* *$ Inferencia: $* * * \mathrm{p}<0.01 ; * * \mathrm{p}<0.05 ; * \mathrm{p}<0.1$

Elaborado: Autores

De los resultados, se observa que el impacto en la nota sigue siendo de 0,54 , mientras, las covariables incorporadas tienen coeficientes que no resultan ser significativos.

\section{Modelo 4}

Para este último modelo, se considera únicamente la covariable escuela con los elementos significativos. 
$y_{i}=\mu+\lambda \cdot T_{i}+\omega \cdot G_{i}+\alpha\left(T_{i} \cdot G_{i}\right)+\beta_{k} X_{k i}+\varepsilon_{i}$ con $\mathrm{k}=3,4,5,6$

De lo cual, $R=0,32021$

Tabla 9: Resultados coeficientes covariables con Modelo 4.

\begin{tabular}{llll}
\hline Covariable(s) & \multicolumn{2}{c}{ Coeff. Error Std.t } & P>t \\
\hline Escuela 3 & -0.479 & 0.167 & -2.870 .004 \\
Escuela 4 & -0.851 & 0.162 & -5.2590 \\
Escuela 5 & 0.958 & 0.172 & 5.5750 \\
Escuela 6 & 1.232 & 0.183 & 6.7350 \\
\hline
\end{tabular}

Tabla 10: Resultados Modelo 4

\begin{tabular}{llllllll}
\hline \multirow{2}{*}{ Variable } & \multicolumn{2}{l}{ Línea Base } & \multicolumn{3}{l}{ Seguimiento } & $\begin{array}{l}\text { Diferencias } \\
\text { en }\end{array}$ \\
\cline { 2 - 6 } & Control & Tratamiento & Diferencia & Control & Tratamiento & Diferencia \\
Nota & 2.956 & 3.51 & 0.553 & 3.971 & 5.062 & 1.091 & 0.538 \\
Std. & 0.161 & 0.162 & 0.156 & 0.161 & 0.162 & 0.156 & 0.22 \\
Error & & & & & & & \\
$\mathrm{t}$ & 18.31 & 6.37 & 3.55 & 9.24 & 7.84 & 4.01 & 2.44 \\
$\mathrm{P}>\mathrm{t}$ & 0 & 0 & $0.000^{* * *}$ & 0 & 0 & $0.000^{* * *}$ & $0.015^{* *}$ \\
\hline
\end{tabular}

* Medias y Errores Estándar son estimados por regresión lineal

$* *$ Inferencia: $* * * \mathrm{p}<0.01 ; * * \mathrm{p}<0.05 ; * \mathrm{p}<0.1$

Elaborado: Autores

El modelo 4 es el mejor se ajusta, teniendo en cuenta que el mismo incorpora las variables significativas. En consecuencia, se puede afirmar con $99 \%$ de confianza que el Tutor Cognitivo tiene impacto de 0,54 sobre la nota promedio.

Para complementar el resultado obtenido en el modelo 4, se calcula el Tamaño del Efecto utilizando la ecuación (3). Con lo cual, se ha obtenido el valor de 0,63, el valor indica que el proyecto de Tutor Cognitivo tiene un efecto alto.

\section{Conclusiones}

- Se pudo establecer que el proyecto Tutor Cognitivo tiene un impacto de 0,54 puntos en la nota promedio de la asignatura de matemática para estudiantes de octavo de básica, con un nivel de confianza del $99 \%$ y un tamaño del efecto alto $(0,63)$. De acuerdo con los modelos estadísticos analizados, se pudo establecer que el modelo que mejor se ajusta, es el que considera la covariable escuela con sus elementos significativos.

- Dado que la educación es un elemento vital para el desarrollo, es necesario ponderarla, dotarle de los mejores recursos y evaluarla permanentemente para determinar objetivamente su evolución e impacto a nivel local, regional, nacional y mundial. 
Destacar que las Tecnologías de la Información y Comunicación (TIC) incorporadas en la educación son una herramienta para alcanzar la calidad educativa.

- De los resultados positivos de la evaluación de impacto al proyecto Tutor Cognitivo, queda la posibilidad de que pueda ser implementado en más instituciones educativas del país. Las futuras intervenciones debieran contemplar un diseño experimental para la evaluación de impacto para complementar los resultados alcanzados en el presente estudio.

\section{Referencias Bibliográficas}

Alcibar, M. F., Monroy, A., \& Jiménez, M. (2018). Impact and use of information and communication technologies in higher education. Información Tecnológica, 29(5), 101110. https://doi.org/10.4067/S0718-07642018000500101

Alderete, M. V., \& Formichella, M. M. (2016). El acceso a las TIC en el hogar y en la escuela: su impacto sobre los logros educativo. Revista de Economía Del Rosario, 19(2), 221-242. Retrieved from https://revistas.urosario.edu.co/index.php/economia/article/view/5626

Asamblea Nacional Constituyente del Ecuador. (2008). Constitución de la República del Ecuador. Retrieved from https://www.asambleanacional.gob.ec/sites/default/files/private/asambleanacional/filesas ambleanacionalnameuid-29/constitucion-republica-inc-sent-cc.pdf

"BBC News Mundo.” (2019). Pruebas PISA: qué países tienen la mejor educación del mundo (y qué lugar ocupa América Latina en la clasificación). BBC News Mundo. Retrieved from https://www.bbc.com/mundo/noticias-internacional-50643441

Espinoza Freire, E., \& Ricaldi Echevarría, L. (2018). El tutor en los entornos virtuales de aprendizaje. Universidad y Sociedad, 10(1), 201-2010. Retrieved from http://scielo.sld.cu/scielo.php?pid=S2218$36202018000300201 \&$ script=sci_arttext\&tlng=en

Fiallos Quinteros, J. C. (2014). FACULTAD LATINOAMERICANA DE CIENCIAS SOCIALES SEDE ECUADOR EVALUACIÓN DE IMPACTO DEL PROYECTO SISTEMA DE TUTORÍA COGNITIVA PARA EDUCACIÓN SECUNDARIA EN ECUADOR. Retrieved from www.flacsoandes.edu.ec

Granados Ospina, A. (2015). Las TIC en la enseñanza de los métodos numéricos. SOFIASOPHIA, 11(2), 143-154.

Hanushek, E. A., Woessmann, L., Ainsworth, M., Benveniste, L., Bourguignon, F., Filmer, D., ... Wößmann, L. (2007). The Role of School Improvement in Economic Development. Retrieved from http://www.nber.org/papers/w12832

Morales, P. (2012). El tamaño del efecto (effect size): análisis complementarios al contraste de medias.

Retrieved

from http://www.upcomillas.es/personal/peter/investigacion/Tama\%F1oDelEfecto.pdf 
“Naciones Unidas.” (2015). Declaración Universal de Derechos Humanos.

Peñaherrera León, M. (2012). Uso de TIC en escuelas públicas de ecuador: análisis, reflexiones y valoraciones. EDUTEC, $16 . \quad$ Retrieved from https://www.edutec.es/revista/index.php/edutec-e/article/view/364/101

Pérez, Á. (2019). Baja calidad de la educación en América Latina. Dinero. Retrieved from https://www.dinero.com/opinion/columnistas/articulo/que-esperaban-de-pisa-2018milagros-por-angel-perez/279879

Secretaría Nacional de Planificación y Desarrollo - SENPLADES. (2009). Plan Nacional de Desarrollo. Retrieved from http://plan.senplades.gov.ec

"Semana." (2017). Informe Unesco sobre educación en América Latina. Semana. Retrieved from https://www.semana.com/nacion/articulo/mision-internacional-de-sabios-entregainforme-que-marcara-la-ruta-educativa-del-pais/643427

Severin, E. (2010). Tecnologías de la información y la comunicación (TICs) en Educación. Marco Conceptual e Indicadores - Banco Interamericano de Desarrollo. División de Educación.

Retrieved from https://publications.iadb.org/publications/spanish/document/Tecnologías-de-lainformación-y-la-comunicación-(TICs)-en-educación.pdf 


\section{PARA CITAR EL ARTÍCULO INDEXADO.}

Villacrés Cáceres, O., Fiallos Quinteros, J. C., Mullo Guaminga, H., \& Diaz Andrade, S. (2020). Evaluación educacional a las tecnologías de información y comunicación con modelos estadísticos. ConcienciaDigital, $3(3.1)$

229-242.

https://doi.org/10.33262/concienciadigital.v3i3.1.1384

\section{¿Ciencia}

El artículo que se publica es de exclusiva responsabilidad de los autores y no necesariamente reflejan el pensamiento de la Revista Conciencia Digital.

El artículo queda en propiedad de la revista y, por tanto, su publicación parcial y/o total en otro medio tiene que ser autorizado por el director de la Revista Conciencia Digital.

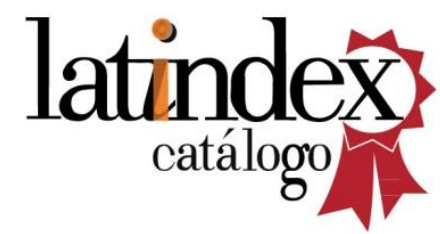

